\title{
On Learning Disentangled Representation for Acoustic Event Detection
}

\author{
Lijian $\mathrm{Gao}^{1}$, Qirong Mao ${ }^{1 *}$, Ming Dong $^{2 *}, \mathrm{Yu} \mathrm{Jing}^{2}$, Ratna Chinnam ${ }^{3}$ \\ *Corresponding authors: mao_qr@ujs.edu.cn, mdong@wayne.edu \\ ${ }^{1}$ School of Computer Science and Communication Engineering, Jiangsu University, Zhenjiang 212013, China \\ ${ }^{2}$ Department of Computer Science, Wayne State University, Michigan 48202, USA \\ ${ }^{3}$ Department of Industrial and Systems Engineering, Wayne State University, Michigan 48202, USA
}

\begin{abstract}
Polyphonic Acoustic Event Detection (AED) is a challenging task as the sounds are mixed with the signals from different events, and the features extracted from the mixture do not match well with features calculated from sounds in isolation, leading to suboptimal AED performance. In this paper, we propose a supervised $\beta$-VAE model for AED, which adds a novel event-specific disentangling loss in the objective function of disentangled learning. By incorporating either latent factor blocks or latent attention in disentangling, supervised $\beta$-VAE learns a set of discriminative features for each event. Extensive experiments on benchmark datasets show that our approach outperforms the current state-of-the-arts (top-1 performers in the Detection and Classification of Acoustic Scenes and Events (DCASE) 2017 AED challenge). Supervised $\beta$-VAE has great success in challenging AED tasks with a large variety of events and imbalanced data.
\end{abstract}

\section{CCS CONCEPTS}

- Computing methodologies $\rightarrow$ Learning latent representations; Feature selection.

\section{KEYWORDS}

acoustic event detection, disentangled latent representation, supervised variational autoencoder

\section{ACM Reference Format:}

Lijian Gao, Qirong Mao, Ming Dong, Yu Jing, \& Ratna Chinnam. 2019. On Learning Disentangled Representation for Acoustic Event Detection In Proceedings of the 27th ACM International Conference on Multimedia (MM'19), October. 21-25, 2019, Nice, France. ACM, NY, NY, USA, 9 pages.

https://doi.org/10.1145/3343031.3351086

\section{INTRODUCTION}

Acoustic event, also known as sound event, is the audio segment that humans label as a distinctive concept in an acoustic signal [3]. The aim of Acoustic Event Detection (AED) is to recognize the sound events presented in a continuous acoustic signal. AED can

Permission to make digital or hard copies of all or part of this work for personal or classroom use is granted without fee provided that copies are not made or distributed for profit or commercial advantage and that copies bear this notice and the full citation on the first page. Copyrights for components of this work owned by others than ACM must be honored. Abstracting with credit is permitted. To copy otherwise, or republish, to post on servers or to redistribute to lists, requires prior specific permission and/or a fee. Request permissions from permissions@acm.org.

MM '19, October 21-25, 2019, Nice, France

(c) 2019 Association for Computing Machinery.

ACM ISBN 978-1-4503-6889-6/19/10 ..\$15.00

https://doi.org/10.1145/3343031.3351086 be used in many applications, such as acoustic surveillance [37], environmental context detection [6], automatic audio indexing [14], etc. In general, there are two types of AED: monophonic detection and polyphonic detection. Monophonic detection deals with the most prominent event at a time instance, while polyphonic detection tackles the situation where multiple sound events happen simultaneously [3].

Speech signals produced in real life are mostly polyphonic. Polyphonic event detection is typically much more complicated than its monophonic counterpart as the sounds are mixed with the signals from different events, and the features extracted from the mixture do not match well with features calculated from sounds in isolation [30]. Moreover, the number of sources emitting at any given moment (polyphony) is unknown and potentially large. Thus, polyphonic AED is highly challenging.

Traditional approaches for polyphonic AED mainly imitate speech recognition and template matching, for example, using combined Gaussian mixture and hidden Markov models [15, 28] with Mel Frequency Cepstral Coefficients (MFCCs) or using non-negative matrix factorization methods $[23,32]$ to represent each sound, and then matching it with a sound dictionary $[9,18]$. However, it is unclear if these hand-tuned features in the traditional methods can sufficiently characterize different sound events. More recently, Deep Neural Networks (DNN) with a bottleneck layer in the middle have been introduced to learn bottleneck features for polyphonic AED and achieved state-of-the-art results [29]. In [3], a multi-label DNN was introduced as the baseline model for the Detection and Classification of Acoustic Scenes and Events (DCASE) challenge [27], which is an official IEEE audio and acoustic signal processing challenge. Approaches based on Long Short-Term Memory (LSTM) networks were also proposed for AED [30] as acoustic signals are temporal and sequential, and LSTM can learn contextual information very well [13].

Although new advances have been made in polyphonic AED through feature learning, how to learn a set of discriminative features by disentangling factors of variation, a very important aspect of AED in real-world environments, is not addressed by current approaches. Unsupervised disentangled representation learning, such as Variational Autoencoder (VAE) [19, 20], $\beta$-VAE [16], InfoGAN [4], and DS-Autoencoder [24], achieved outstanding performance at capturing the generative factors in data. However, if applied directly to polyphonic AED, the same set of features is learned to detect all the events in a given audio segment, which could lead to degenerated performance, especially when the number of target events is large, e.g., greater than 10 [26]. That is, the feature set is not sufficiently expressive for a large variety of events. 
In this paper, we propose a supervised $\beta$-VAE model by adding a novel event-specific disentangling loss in the objective function of disentangled learning. Our model consists of an encoder, a disentangling layer and a decoder. By incorporating either latent factor blocks or latent attention mechanism in the disentangling layer, supervised $\beta$-VAE learns a set of discriminative features for each event and thus can handle a large variety of events in polyphonic AED. The major contribution of our work is summarized as follows.

1. To the best of our knowledge, this is the first work that introduces disentangled feature representation to polyphonic AED. Compared with AED in a controlled environment with very limited number of event types, this work addresses AED challenges in the real-world scenario, potentially with a large variety of events. Another advantage of introducing a generative model into AED is that we can generate more training data for a given event to improve detection accuracy through data augmentation.

2. We propose a novel event-specific disentangling loss to learn a discriminative latent representation for each acoustic event. In our model, a disentangling layer is designed accordingly through either latent factor blocks and latent attentions.

3. Extensive experiments on benchmark datasets show that our approach significantly outperforms the current state-of-thearts (top-1 performers in the DCASE 2017 AED challenge) Our model has great success with challenging real-world AED datasets, recorded in natural environments with a large variety of events and imbalanced data for different target events.

The rest of the paper is organized as follows. We introduce the related work in Section 2 . Section 3 presents the architecture and algorithm of supervised $\beta$-VAE in details. Section 4 describes the experiment setup and the performance evaluation. Conclusion is given in Section 5.

\section{RELATED WORK}

\subsection{Feature learning in polyphonic AED}

In the field of polyphonic AED, research mainly focuses on learning better features to represent polyphonic sound events from raw acoustic features. The most popular acoustic features are Mel Frequency Cepstral Coefficients (MFCCs) [8], which are representations of the short-term power spectrum of sound, based on a linear cosine transform of a log power spectrum on a nonlinear mel scale of frequency. MFCCs achieved good performance for AED in the past years $[15,28]$, but was recently outperformed by deeply learned features. It was shown that DNN can better learn a feature representation by mapping the raw features into a higher dimensional space and then reducing the dimension to remove the redundant features, which help achieve a significant boost in classification performance when being applied to AED [11]. DNNs are the most popular models in the DCASE 2016 challenge, and also became the baseline model for DCASE 2017 challenge. Moreover, the learned bottleneck features from DNN were also combined with MFCCs for AED in [29].

Long Short-Term Memory (LSTM) was also recently adopted in AED to explicitly model the temporal context. LSTM is one of the most widely used recurrent structures in sequence modeling, which aims to use gates to control information flow in the recurrent computation [25]. Different from DNN, LSTM can directly model the sequential information that is naturally presented in audio so that the temporal structure is learned simultaneously with deep features. Models with bidirectional and unidirectional recurrence were both shown to work well for AED [34, 35, 38]. For example, in [30] a multi-label bi-directional LSTM was introduced into polyphonic AED and archived solid performance.

\subsection{Disentangled representation}

Disentangled representation can be defined as one where single latent units are sensitive to changes in single generative factors, while being relatively invariant to changes in other factors [2]. For example, a model trained on a dataset of 3D objects might learn independent latent units sensitive to single independent data generative factors such as object identity, position, scale, lighting or colour, thus acting as an inverse graphics model [22].

Initially, most disentangled representation methods require the prior knowledge of the number and/or nature of the data generative factors $[5,31,36]$, which is not always available, especially in the real world applications, where the data generative factors are typically not clearly defined. Later, unsupervised disentangled representation was proposed and achieved good performance [7]. InfoGAN [4] extends the Generative Adversarial Network (GAN) [12] to additionally maximise the mutual information between a subset of the generating noise variables and the output of a recognition network. It was reported to be capable of discovering at least a subset of data generative factors and of learning a disentangled representation of these factors [16]. However, the training of the InfoGAN is unstable.

A more stable method, named $\beta$-VAE [16], augments the original VAE framework with a single hyperparameter $\beta$ that modulates the learning constraints applied to the model. With $\beta>1$, the model is pushed to learn a more efficient latent representation of the data and achieves outstanding performance in image generation. $\beta$ VAE is a variant of variational autoencoder. VAE infers $\log p_{\theta}(x)$, the marginal $(\log )$-likelihood distribution of data $x$. As $p_{\theta}(x)$ is intractable, variational lower bound is derived as,

$$
\begin{aligned}
& \log p_{\theta}(x) \geq \mathcal{L}(\theta, \phi ; x) \\
& =\mathbb{E}_{q_{\phi}(z \mid x)}\left[\log p_{\theta}(x \mid z)\right]-\beta D_{K L}\left(q_{\phi}(z \mid x) \| p(z)\right)
\end{aligned}
$$

where $q_{\phi}(z \mid x)$ is the probabilistic approximation to the true intractable posterior density $p_{\theta}(z \mid x) . z \in R^{M}$ are the underlying factors to generate data $x$, and $q_{\phi}(z \mid x)$ can be learned through an encoding neural network. When $\beta=1$, the model is reduced to VAE. By setting $\beta>1$, posterior distribution $q_{\phi}(z \mid x)$ is forced to extract disentangled features as $q_{\phi}(z \mid x)$ is encouraged to match with a prior $p(z)$ which is a unit Gaussian $(p(z)=\mathcal{N}(0, I))$.

\section{SUPERVISED $\beta$-VAE FOR POLYPHONIC AED}

In this section, we propose a supervised $\beta$-VAE model to learn event-specific disentangled representation for polyphonic AED. Our model consists of an encoder, a disentangling layer and a decoder. Inspired by the idea of feature disentanglement, we assume there exist latent variables that control presence of acoustic events and design the disentangling layer to directly reveal the underlying 
indicative latent factors for each sound event. Next, we discuss our model in details.

\subsection{Supervised $\beta$-VAE}

Supervised $\beta$-VAE aims to decompose event-specific latent representation $p_{\theta}\left(z^{*} \mid x\right)$ from raw acoustic features, which are crucial for accurate sound event detection. Specifically, it takes MFCCs as input $x$, encodes them into event-specific low-dimensional generative factors $z$ and then reconstructs MFCCs by passing $z$ through a decoder, in the meanwhile, it learns $z^{*}$ from $z$ in the disentangling layer. Different from unsupervised $\beta$-VAE, our method also aligns an event detector to each of $z_{k}^{*}$ to predict presence of a specific sound event.

Let $z^{*}=\left\{z_{1}^{*}, z_{2}^{*}, \ldots, z_{k}^{*}, \ldots, z_{K}^{*}\right\}$ be the class of latent factors, where each $z_{k}^{*}$ is a subset of latent variables that controls presence of the $k$-th sound event, and $K$ is the total number of events. The objective of supervised $\beta$-VAE is:

$$
\max _{\theta} \mathbb{E}_{p_{\theta}\left(z^{*}\right)}\left[\log p_{\theta}\left(x \mid z^{*}\right)\right]
$$

Following [16], we derive variational lower bound on inferring marginal (log-)likelihood,

$$
\begin{aligned}
& \mathbb{E}_{p_{\theta}\left(z^{*}\right)}\left[\log p_{\theta}\left(x \mid z^{*}\right)\right] \\
& \geq \mathbb{E}_{q_{\phi}\left(z^{*} \mid x\right)}\left[\log p_{\theta}\left(x \mid z^{*}\right)\right]-\beta D_{K L}\left(q_{\phi}\left(z^{*} \mid x\right) \| p_{\theta}\left(z^{*}\right)\right)
\end{aligned}
$$

where $p\left(z^{*}\right)$ is the joint distribution of all subsets of event-specific latent variables, $p\left(z^{*}\right)=p\left(z_{1}^{*}, z_{2}^{*}, \ldots, z_{K}^{*}\right)$.

We assume there exist $K$ independent sets of latent variables, each of which controls the variation of an sound event. Then, KullbackâĂŞLeibler divergence term in the RHS of Eq. 3 equals to,

$$
\begin{aligned}
& D_{K L}\left(q_{\phi}\left(z^{*} \mid x\right) \| p_{\theta}\left(z^{*}\right)\right) \\
& =\sum_{k=1}^{K} D_{K L}\left(q_{\phi}\left(z_{k}^{*} \mid x\right) \| p_{\theta}\left(z_{k}^{*}\right)\right) \\
& =\sum_{k=1}^{K} \frac{1}{2 I} \sum_{i=1}^{I}\left[\left(\mu_{k}^{*(i)}\right)^{2}+\left(\sigma_{k}^{*(i)}\right)^{2}\right. \\
& \left.-1-\log \left(\sigma_{k}^{*(i)}\right)^{2}\right] \\
& :=\sum_{k=1}^{K} D_{K L}^{*(k)}\left(\phi ; x, z^{*}\right)
\end{aligned}
$$

where $i$ denotes the $i$-th training sample; $\mu_{k}^{*(i)}$ and $\sigma_{k}^{*(i)}$ are the mean and variance of $z_{k}^{*(i)}$, respectively. For each latent representation $z_{k}^{*}$, the inferred posterior configuration $q_{\phi}\left(z_{k}^{*} \mid x\right)$ should also be matched to the prior $p\left(z_{k}^{*}\right)$, where $p\left(z_{k}^{*}\right)=\mathcal{N}(0, I)$.

In supervised $\beta$-VAE, a event detector is aligned with each $z_{k}^{*}$, which contains a multilayer perceptron with a sigmoid layer as the output. All $K$ detectors are trained by optimizing the binary cross entropy loss,

$$
\begin{array}{r}
\mathcal{L}_{A E D}^{(k)}\left(\theta^{\prime} ; y\right)=-\sum_{i=1}^{I}\left[y_{k}^{(i)} \log \left(p\left(y_{k}^{(i)}\right)\right)\right. \\
\left.+\left(1-y_{k}^{(i)}\right) \log \left(1-p\left(y_{k}^{(i)}\right)\right)\right]
\end{array}
$$

where $y_{k}^{(i)}$ denotes the ground truth of the $i$-th sample, either 1 or $0 ; p\left(y_{k}^{(i)}\right)$ is the probability of the $i$-th sample detected as the $k$-th event; and $I$ is the total number of samples.

By joining the detection loss $\mathcal{L}_{A E D}^{(k)}$ in Eq. 5 and the modified Kullback-Leibler divergence $D_{K L}^{*(k)}$ in Eq. 4 , we propose a novel event-specific disentangling loss as follows,

$$
\begin{array}{r}
\mathcal{L}_{\text {disent }}\left(\phi, \theta^{\prime} ; x, y, z\right)=\frac{1}{K} \sum_{k=1}^{K}\left[\beta D_{K L}^{*(k)}\left(\phi ; x, z^{*}\right)\right. \\
\left.+\mathcal{L}_{A E D}^{(k)}\left(\theta^{\prime} ; y\right)\right]
\end{array}
$$

where the hyper-parameter $\beta$ weighs the degree of disentanglement in the latent representation for each sound event. Putting all the components of the loss function in the disentangling layer and the decoder together, supervised $\beta$-VAE is trained in an end-to-end manner with the following loss,

$$
\begin{array}{r}
\mathcal{L}_{s-\beta-V A E}\left(\theta, \phi, \theta^{\prime} ; x, y, z\right)=\mathcal{L}_{\text {recons }}(\theta, \phi ; x, z) \\
\left.+\lambda \mathcal{L}_{\text {disent }}\left(\phi, \theta^{\prime} ; x, y, z\right)\right)
\end{array}
$$

where $\mathcal{L}_{\text {recons }}(\theta, \phi ; x, z):=\mathbb{E}_{q_{\phi}\left(z^{*} \mid x\right)}\left[\log p_{\theta}\left(x \mid z^{*}\right)\right]$ is the reconstruction loss, which can take the form of either binary cross entropy or mean squared error, and the $\lambda$ weights the contribution of event detection and reconstruction.

\subsection{Architecture of supervised $\beta$-VAE}

The architecture of the proposed supervised $\beta$-VAE model is shown in Fig.1, which has an encoder, a disentangling layer and a decoder. Encoder: Our encoder is similar to the encoder in unsupervised $\beta$-VAE. We design two different kinds of encoders: DNN and LSTM, to model a single frame or a sequence of frames, respectively. We first extract the MFCCs $X$ from the sound signal $S$. The sound signal is denoted as $S=S(t)$ if the encoder is DNN or $S=[S(t-$ $n), \ldots, S(t)]$ if the encoder is LSTM, where $t$ represents the time, and $n$ stands for time steps. Then, $X$ is compressed into a low-dimensional Gaussian space with mean $\mu$ and variance $\sigma$ by the encoder. Here, $\mu$ and $\sigma$ are two feature vectors with the same dimension. With the reparameterization trick, we get the latent representation $z$ $(z=\mu+\sigma \odot \varepsilon)$ of the current frame, where $\varepsilon \sim \mathcal{N}(0,1)$ is a random number. Note that $z$ also contains the temporal information for a sequential input. The goal of encoder is to reveal the generative factors $z$.

Disentangling Layer: Disentangling layer is the core component of our model. As mentioned above, latent representation $\mu(x)$ and $\sigma(x)$ from encoder is not directly related to sound event detection. Here, the disentangling layer is designed to learn event-specific latent representation $z^{*}$ based on the latent factors $z$ obtained by the encoder. To this end, we propose two methods: latent factor blocks and latent attention that are detailed as follows.

(a) Latent factor blocks: we partition $z$ into $K$ latent factor blocks, where each block $z_{k}^{*}$ has $\frac{M}{K}$ dimension. That is, $z_{k}^{*}=z\left[\frac{(k-1) M}{K}\right.$ : $\left.\frac{k M}{K}\right]$. We force the model to learn the event-specific disentangled factors and get the most efficient latent representation for each sound event.

(b) Latent attention: another strategy is using attention mechanism to weight the latent factors $z$, which provides more flexibility 


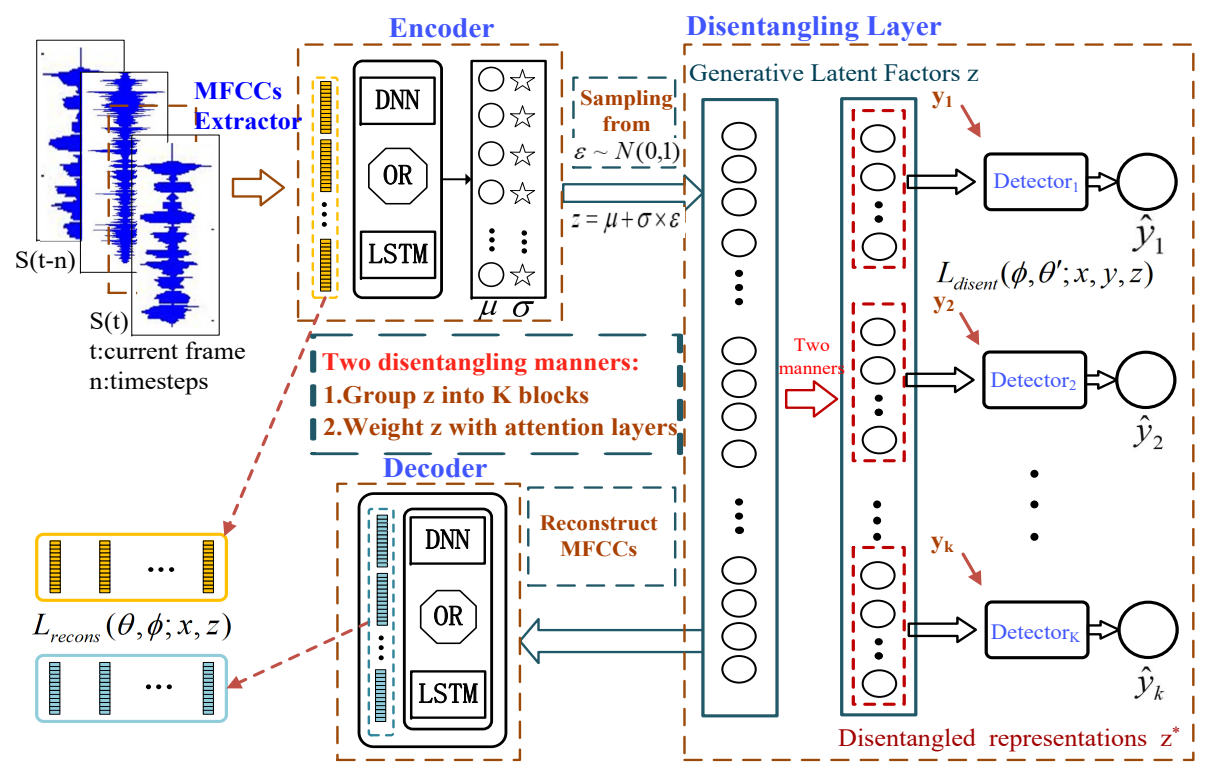

Figure 1: The architecture of supervised $\beta$-VAE for AED.

to our model by avoiding the limitation of encoding the input sequence to one fixed-length latent vector. The latent factors $z$ are connected to $K$ full-connected attention layers separately. One such layer is shown in Fig.2, in which after activated by softmax, we gain the $k$ th layer attention weights of the latent factors $z$ for each sound event,

$$
a_{k}=\operatorname{softmax}_{k}(z)
$$

where $a_{k}$ is a vector that has the same dimension as $z$, and $k \leq K$. Then, the latent representation $z_{k}^{*}$ for each sound event is given by:

$$
z_{k}^{*}=a_{k} * z
$$

Decoder: To ensure that the latent space captures data generative factors while discarding the noise, it is important to train a decoder to reconstruct the given input. Here, the decoder takes the same model as the encoder (DNN or LSTM) to maintain the symmetry of the network structure.

The detailed algorithm for Supervised $\beta$-VAE for AED is given in Algorithm 1.

\section{EXPERIMENTS}

\subsection{Datasets}

We choose two widely-adopted AED benchmark datasets for experimental evaluations: TUT2017 [27], the Tampere University of Technology sound event 2017 dataset, provided by the DCASE 2017 challenge, and Freesound database [10], a public sound event dataset harvested from Freesound.

TUT 2017: Every year, the DCASE challenge has different tasks in the field of AED. For example, in 2018, the AED task is "large-scale

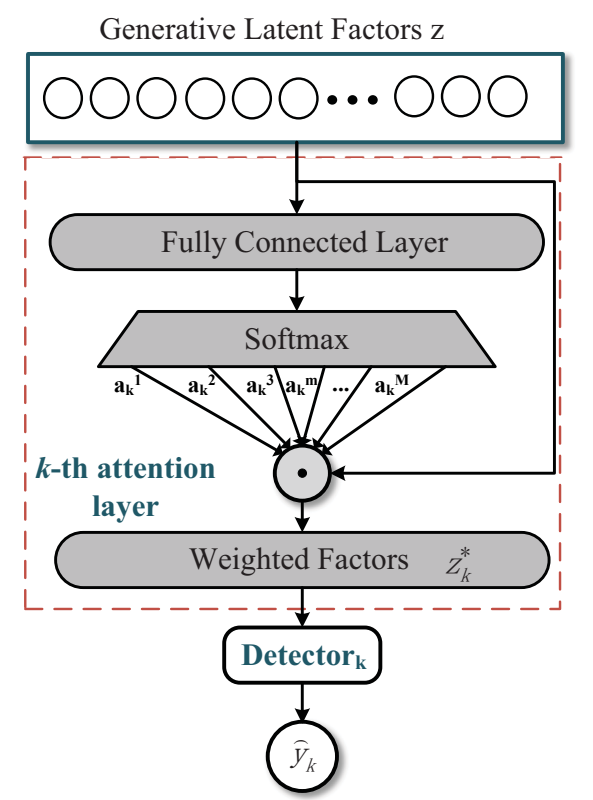

Figure 2: Latent attention in the disentangling layer.

weakly labeled semi-supervised sound event detection in domestic environments", and in 2017, it is "Sound event detection in real life audio". Clearly, the topic of DCASE 2017 challenge matches nicely with the research in this paper. Thus, we select TUT 2017 dataset used in DCASE 2017 AED challenge in our experimental 


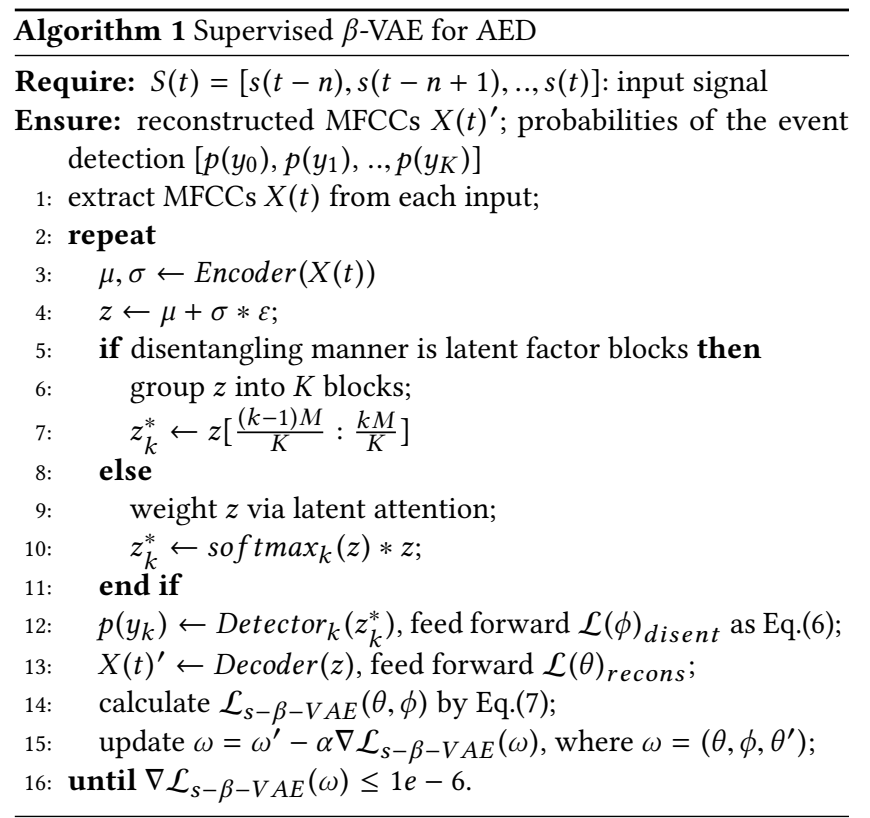

evaluation. TUT 2017 is a polyphonic sound event dataset consisting of recordings of street acoustic scenes with various levels of traffic and other activities. The scenes were selected as representing an environment of interest for detection of sound events related to human activities and hazard situations. There are six types of events included in this dataset: brakes squeaking, car, children, large vehicle, people speaking, and people walking. The lengths of the recordings given in seconds are 67.6, 2541.5, 346.1, 727.0, 630.6, and 1079.2 , respectively.

Freesound: The Freesound dataset is a new monophonic acoustic event database harvested from Freesound, a repository of audio samples uploaded by users, which consists of 28 types of events. Note that, since there are no public AED datasets that contain data with different polyphony levels, here we construct new datasets from Freesound dataset by randomly mixing the sound signals from Freesound. Specifically, one polyphonic signal $s^{\prime}$ can be constructed by using several monophonic signals $s_{p}$ as follows,

$$
s^{\prime}=\frac{1}{P} \sum_{p=0}^{P}\left(s_{p}\right)+\text { noise }
$$

where $p \leq P$ and $P$ is the number of event categories (also known as the polyphony level), and noise denotes stochastic noise. We construct four new polyphonic datasets with increasing polyphony levels (from 5 to 20 with a step of 5), and each of them contains 2000 mixed sound signals. These datasets are used to evaluate the performance of our model in environments with gradually increasing complexity.

\subsection{Experiment setup}

In our experiments, we evaluated supervised $\beta$-VAE using the same setting as in the DCASE 2017 challenge.
(1) We use MFCCs as inputs to train a three-layer DNN, whose output layer is activated by sigmoid units. To avoid overfitting, each fully connected layer was followed by a Dropout layer with a 0.5 dropout rate.

(2) All the experiments were conducted with four-fold crossvalidation, where a training dataset is divided into four-folds, three for training and one for validation.

(3) In all the experiments, we used two well-accepted metric of polyphonic AED: segment-based F1 score and Error Rate (ER) to measure the performance of various methods. Both metrics are calculated with one-second-long segments as follows,

$$
F 1=\frac{2 \cdot \sum_{k=1}^{K} T P(k)}{2 \cdot \sum_{k=1}^{K} T P(k)+\sum_{k=1}^{K} F P(k)+\sum_{k=1}^{K} F N(k)}
$$

where for the segment $k, T P(k)$ is the true positives, $F P(k)$ is the false positives, and $F N(k)$ is the false negatives.

$$
E R=\frac{\sum_{k=1}^{K} S(k)+\sum_{k=1}^{K} D(k)+\sum_{k=1}^{K} I(k)}{\sum_{k=1}^{K} N(k)}
$$

where $N(k)$ is the total number of active sound events in the ground truth of the segment $k$, and $S(k), D(k)$ and $I(k)$ are the number of substitutions, deletions and insertions, respectively.

To determine the best architecture for supervised $\beta$-VAE, we first performed ablation study to choose the model for the encoder, the decoder, and the disentangling layer. Specifically, the encoder and decoder are selected from either DNN or LSTM, and the disentangling layer is evaluated between latent factors blocks and latent attention. Then, we compared our model with the baseline models (DNN and LSTM) and the state-of-the-arts, the top-1 F1 model: J-NEAT [21] and the top-1 ER model: CRNN [1] in the DCASE 2017 AED challenge. Specifically, J-NEAT is a co-evolution extension of the neuro-evolution and augmenting topology algorithm, which evolves artificial neural networks using genetic algorithms. CRNN is one of the most popular models in polyphonic AED, which has a multichannel convolutional and recurrent neural network architecture.

All the experiments are conducted with Ubuntu 16.04 LST, Python 3.6.2 with Keras 2.2.0 and TensorFlow 1.8.0 on a NVIDIA TITAN X GPU. We performed a simple grid search to choose the hyperparameters $\beta$ in Eq.6 (integers in [3,5]) and $\lambda$ in Eq.7 (integers in $[1,3]$ ). For the TUT dataset, $\beta$ and $\lambda$ are set to 4 and 1, respectively. For the Freesound dataset, they are set to 3 and 1 for 5 events, 4 and 2 for 10 events, 5 and 3 for 15 events, and 5 and 2 for 20 events, respectively. The batch size and epochs in the training are both empirically set at 200. The complete source code for our implementation is available in the supplemental materials, which we will make publicly available through Github together with our datasets after the double-blind review period.

\subsection{Model ablation}

We performed model ablation on the TUT dataset. To choose the best architecture, we first use two kinds of encoder/decoder (DNN and LSTM) to train an unsupervised $\beta$-VAE, and then use the learned latent representation to train a multi-label event detector. The 
Table 1: F1 scores on TUT dataset with different encoder/decoder. DNN has three fully connected layers with 50 units followed by Dropout layers. The Drop-Connected LSTM [33] has two layers with 5 cells (which means the time step is 5), each of which has $\mathbf{5 0}$ units followed by dropconnect layers (the same basic setup with DNN). Detector consists of two fully connected layers with 32 and 6 units, respectively. Latent denotes the number of latent variables. The highest score is highlighted in bold. Detector and Latent are used similarly in all the tables in the experiment section.

\begin{tabular}{ccc}
\hline Model & Parameters & F1(\%) \\
\hline \multicolumn{3}{c}{ Encoder(DNN) } \\
$\beta$-VAE(DNN) & Lecotent(15) & 42.53 \\
& Detector(32, (6,'sigmoid')) \\
\hline \multicolumn{3}{c}{ Encoder(LSTM) } \\
$\beta$-VAE(LSTM) & Latent(15) & $\mathbf{4 3 . 9 0}$ \\
& Decoder (LSTM) & \\
\hline
\end{tabular}

Table 2: F1 scores on the TUT dataset with different disentangling methods: latent block and latent attention. Block indicates the number of latent factors in each block, and Att indicates the same. The highest score is highlighted in bold.

\begin{tabular}{ccc}
\hline Model & Parameters & F1(\%) \\
\hline & Encoder $($ LSTM $)$ & \\
& Latent $(15 \times 6)$ & \\
s- $\beta$-VAE(LSTM)-block & Decoder $($ LSTM $)$ & 45.24 \\
& $6^{*}$ Block $(15)$ & \\
& $6^{*}$ Detector $(32,(6$, 'sigmoid')) \\
\hline & Encoder $($ LSTM $)$ \\
s- $\beta$-VAE(LSTM)-attention & Latent $(15)$ \\
& Decoder $($ LSTM $)$ & $\mathbf{4 5 . 8 6}$ \\
& $6^{*}$ Att $(15$, 'softmax') \\
& $6^{*}$ Detector $(32,(6$, 'sigmoid')) \\
\hline
\end{tabular}

$F 1$ scores obtained by the unsupervised $\beta$-VAE with different encoder/decoder are shown in Table 1. Clearly, the LSTM network achieves better performance. Thus, we select the LSTM network as the encoder/decoder in our following experiments.

In order to select the best disentangling method, we compared the $F 1$ scores of supervised $\beta$-VAE with latent factors blocks and latent attentions. As seen from Table 2, the attention-based supervised $\beta$-VAE outperforms the block-based one. Thus, latent attention is selected as the disentangling method in the remaining experiments. Also note that both $F 1$ scores obtained by our supervised $\beta$-VAE models (Table 2) are higher than that of the (original) unsupervised $\beta$-VAE (Table 1).

\subsection{AED performance evaluation}

TUT in the DCASE 2017 AED challenge: We first report the F1 and ER scores on the TUT 2017 dataset obtained by our model, the baseline models (DNN \& LSTM), and two top-1 performers in
Table 3: F1 and ER comparison among our model, the baseline models and two top-1 performers on the TUT 2017 dataset. Dense is a fully connected layer while $L S T M$ is one layer LSTM network.

\begin{tabular}{|c|c|c|c|}
\hline Model & Parameters & F1(\%) & ER \\
\hline DNN & $\begin{array}{c}\text { Dense(50, 'Relu') } \\
\text { Dense(50, 'Relu') } \\
\text { Dense(6, 'sigmoid') }\end{array}$ & 42.80 & 0.9358 \\
\hline LSTM & $\begin{array}{c}\operatorname{LSTM}(50, \text { 'tanh') } \\
\operatorname{LSTM}(50, \text { 'tanh') } \\
\operatorname{Dense}(6, \text { 'sigmoid') }\end{array}$ & 43.22 & 0.9031 \\
\hline $\begin{array}{c}\text { J-NEAT } \\
\text { (DCASE2017 TOP-1 F1) }\end{array}$ & {$[21]$} & 44.90 & 0.8979 \\
\hline $\begin{array}{c}\text { CRNN } \\
\text { (DCASE2017 TOP-1 ER) }\end{array}$ & {$[1]$} & 41.70 & 0.7914 \\
\hline $\mathrm{s}-\beta$-VAE(LSTM)-attention & $\begin{array}{c}\text { Encoder }(\text { LSTM }) \\
\text { Latents }(15) \\
\text { Decoder }(\text { LSTM }) \\
6^{*} \text { Att }(15, \text { 'softmax') } \\
6^{*} \text { Detector }(32,(6, \text { 'sigmoid')) }\end{array}$ & 45.86 & 0.8259 \\
\hline
\end{tabular}

the DCASE 2017 AED challenge. The results of J-NEAT and CRNN models are directly obtained from the leaderboard ${ }^{1}$ of the DCASE 2017 AED challenge. As shown in Table 3, our attention-based supervised $\beta$-VAE achieved the best F1 score while maintaining a very competitive ER (only slighter higher than CRNN). Actually, as seen from the leaderboard, it is very difficult to obtain competitive performance on both F1 score and ER for the same method. For example, the top-1 F1 model J-NEAT got the best F1 score (44.9\%) while its ER ranked 15th. The top-1 ER model CRNN achieved the best ER (0.7914), but its F1 score ranked 11th. As a comparison, our attention-based supervised $\beta$-VAE achieved the highest F1 score and would rank fourth based on ER. This clearly demonstrates the effectiveness of our event-specific latent representations.

To gain an intuitive understanding of the learned event-specific latent features, we visualize and compare the distributions of the features learned by DNN and our model, respectively. For DNN, we extract the features from sound samples with single event because DNN features are not learned in an event-specific way, while for our model, we show the features learned from mixed sounds. Figs. 3 (a) and (b) show the feature distributions in 2D for six events in the TUT dataset. Clearly, supervised $\beta$-VAE learns a more discriminative (between events) and compact (within an event) feature representation, ultimately leading to superior AED performance.

Freesound: Here, we evaluate our model on Freesound, more challenging and realistic AED data collected from natural environments. The biggest advantage of our model is its capability to handle real-world AED tasks, potentially with a large variety of events as well as imbalanced data for different target events. This is where other AED methods drastically worsen in performance. First, we compared our method with DNN and CRNN based on both F1 score and ER with an increasing number of event types (from 5 to 20). As shown in Table 4, attention-based supervised $\beta$-VAE clearly achieves the best performance in all cases by a large margin. Note that the F1 score of our model only drops $4.40 \%$ when the number of events increases from 5 to 20 , while CRNN drops $7.28 \%$ and DNN

${ }^{1}$ http://www.cs.tut.fi/sgn/arg/dcase2017/challenge/task-sound-event-detection-inreal-life-audio-results 


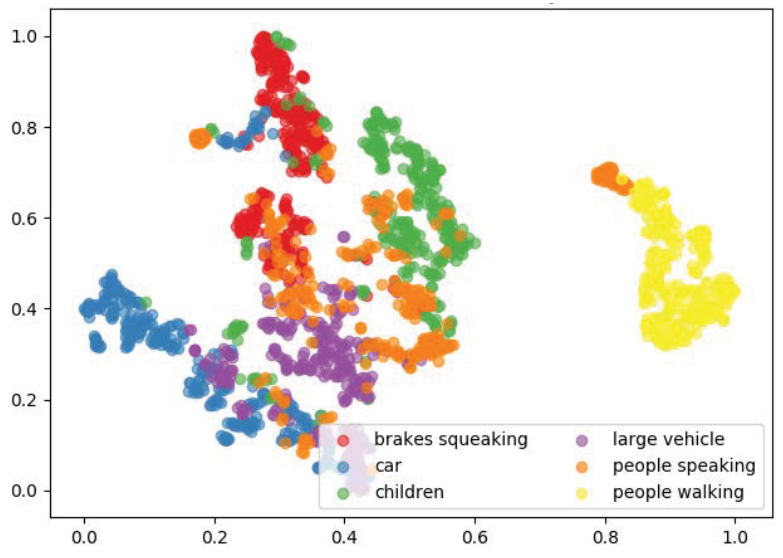

(a) Event feature distribution learned by DNN.

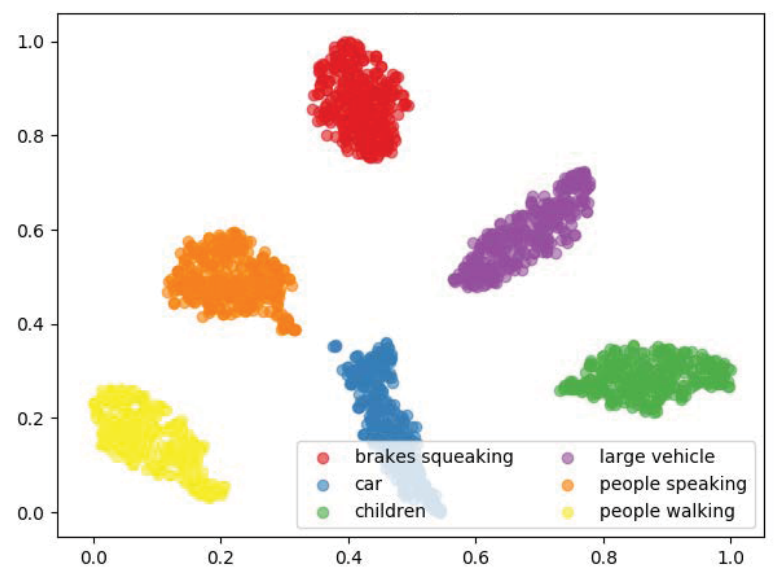

(b) Event feature distribution learned by attention-based supervised $\beta$-VAE.

Figure 3: Feature distributions from DNN and our model. Features are first extracted from the outputs of last hidden layer before the sigmoid layer in event detectors and then mapped using t-SNE [17] to 2D.

declines by $32.75 \%$. The same conclusion can be drawn for ER as well. Recall that CRNN was the top-1 ER method in the DCASE 2017 AED challenge.

Next, we constructed a dataset with imbalance data samples from five sound events including "guitar", "car", "cat", "bird" and "applause", which are randomly selected from the original Freesound dataset. Since supervised $\beta$-VAE is a generative model, we can generate more training data for the events that have less available samples and then re-train our sound detectors, which also shows that our model is capable of disentangling multiple sounds and generate event-specific sound. More specifically, before data augmentation, the total number of training samples of "guitar" is only about half of the other events'. We decoded the latent variables $z_{\text {guitar }}^{*}$ to double the "guitar" samples and then re-trained our model. The AED results reported in Table 5 show that the detection accuracy for "guitar" improves from $67.24 \%$ to $72.49 \%$, resulting in a $3.43 \%$ improvement in F1 scores and a 0.016 reduction in ER. From
Table 4: F1 and ER comparison with different number of events on the Freesound dataset. Number of latent variables are set to $15,30,45,60$ as the event types increase from 5 to 20.

\begin{tabular}{cccc}
\hline No. of events & Model $^{*}$ & F1(\%) & ER \\
\hline \multirow{3}{*}{5} & DNN & 74.14 & 0.4215 \\
& CRNN & 78.58 & 0.3544 \\
& s- $\beta$-VAE(LSTM) & $\mathbf{8 9 . 4 9}$ & $\mathbf{0 . 1 9 5 4}$ \\
\hline \multirow{3}{*}{10} & DNN & 69.26 & 0.4028 \\
& CRNN & 74.48 & 0.2700 \\
& s- $\beta$-VAE(LSTM) & $\mathbf{8 7 . 1 9}$ & $\mathbf{0 . 2 0 0 6}$ \\
\hline \multirow{3}{*}{15} & DNN & 62.77 & 0.5698 \\
& CRNN & 72.86 & 0.3789 \\
& $\mathbf{s}-\beta$-VAE(LSTM) & $\mathbf{8 4 . 5 8}$ & $\mathbf{0 . 2 0 9 6}$ \\
\hline \multirow{3}{*}{20} & DNN & 41.39 & 0.7254 \\
& CRNN & 71.30 & 0.3917 \\
& $\mathbf{s}-\beta$-VAE(LSTM) & $\mathbf{8 5 . 0 9}$ & $\mathbf{0 . 1 9 9 9}$ \\
\hline
\end{tabular}

the results reported in this section, it is clear that our model can handle large event varieties and imbalanced training data. It has great success in AED in natural environments.

\subsection{Qualitative disentanglement visualization}

Finally, we qualitatively show event-specific disentangled factors learned by supervised $\beta$-VAE. Specifically, we alter one latent variable while fixing others and visualize the corresponding changes in the generated data (MFCCs). First, we define a function to quantify the changes as follows,

$$
\nabla(\text { init }, \text { altered })=\mid \operatorname{Decoder}(\text { altered })-\operatorname{Decoder}(\text { init }) \mid
$$

where init and altered denote the initial and altered values of $z_{k}^{*}[m]$, the $m$-th latent variable associated with the $k$-th event, respectively. In Fig.4, we use heat maps to visualize the changes on 250 sound samples from three event categories: "brakes squeaking", "children", and "people speaking" by altering some disentangled latent factors. In each sub-image, the $\mathrm{x}$-axis denotes the number of samples, the $y$-axis denotes the dimension of the MFCC features, and the color shows the normalized magnitude of changes in the range of $[0,1]$, where the red color indicates a bigger change while the blue is for a smaller one.

Some important observations in Fig.4 are highlighted in panels A (red box) and B (blue box). (A) shows $\nabla$ when changing the values of two disentangled latent factors $z_{0}^{*}[6]$ and $z_{0}^{*}[14]$ for the event "brakes squeaking". Note that the values of $z^{*}$ are varied within the bounds of its original range. The horizontal patterns in a sub-image clearly shows that different MFCC features in the sound samples are controlled by different factors in $z^{*}$. For example, the left two images in panel A are obtained by altering $z_{0}^{*}[6]$ from -1.5 to -1 and -1.5 to -0.4 , respectively, and they show similar changes on MFCCs. If we compare the images obtained by altering different factors, e.g., $z_{0}^{*}[6]$ and $z_{0}^{*}[14]$, the changes on MFCCs are clearly different. So, different disentangled factors control different dimensions in the output for the same event. 
Table 5: F1 scores and ER before and after data augmentation for the "guitar" event.

\begin{tabular}{|c|c|c|c|c|c|c|c|}
\hline \multirow{2}{*}{$\begin{array}{c}\text { Data } \\
\text { augmentation }\end{array}$} & \multicolumn{5}{|c|}{ Accuracy of events detection(\%) } & \multirow{2}{*}{ F1(\%) } & \multirow{2}{*}{ ER } \\
\hline & guitar & car & cat & bird & applause & & \\
\hline Before & 67.24 & 79.02 & 76.78 & 71.61 & 77.24 & 75.27 & 0.4121 \\
\hline After & 72.49 & 78.13 & 77.40 & 72.38 & 77.41 & 78.70 & 0.3861 \\
\hline
\end{tabular}

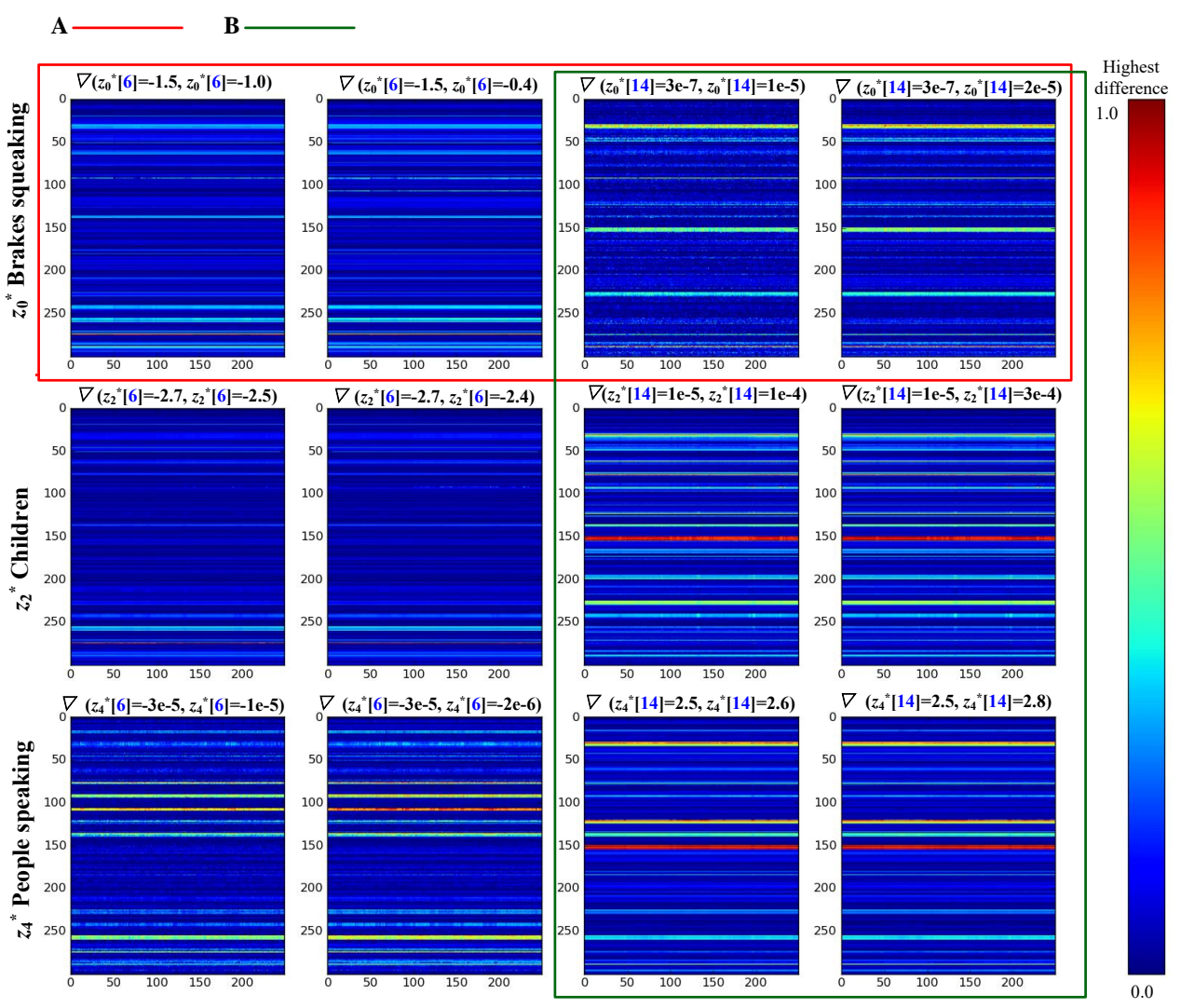

Figure 4: Disentanglement visualization.

(B) shows $\nabla$ when changing the values of the same disentangled latent factors $z^{*}[14]$ for the three events "brake squeaking", "children" and "people speaking". Clearly, $z^{*}[14]$ controls different MFCCs associated with each event, and the changes of its value result in changes in different regions of the sub-images. Recall that the latent attention layer in supervised $\beta$-VAE assigns an independent set of weights to $z$ for each event. Thus, through latent attention, the same disentangled factor gains the flexibility to control different dimensions in the output for different events. The effectiveness of event-specific disentangled learning for AED is clearly demonstrated by these qualitative results.

\section{CONCLUSION AND FUTURE WORK}

The aim of AED is to recognize the sound events presented in a continuous acoustic signal. In this paper, we propose a novel model, supervised $\beta$-VAE, to learn disentangled feature representation for polyphonic AED. In supervised $\beta$-VAE, we added an event-specific disentangling term in the loss function, which is implemented through latent factor blocks or latent attention in the disentangling layer of our model. Compared with current state-of-the-arts, our approach achieved superior performance on challenging polyphonic AED tasks.

Even though supervised $\beta$-VAE is designed for AED, it potentially provides a general framework for acoustic detection and recognition. In the future, we plan to extend supervised $\beta$-VAE and apply it to other important tasks in audio processing, such as speaker recognition in a mixed sound.

\section{ACKNOWLEDGEMENT}

This work was supported by National Natural Science Foundation of China Grants 61672267 and U1836220 (to Q.M) and US National Science Foundation (NSF) grant CNS-1637312 (to M.D). 


\section{REFERENCES}

[1] Sharath Adavanne and Tuomas Virtanen. 2017. A Report on Sound Event Detection with Different Binaural Features. Technical Report. DCASE2017 Challenge.

[2] Y Bengio, A Courville, and P Vincent. 2013. Representation learning: a review and new perspectives. IEEE Trans Pattern Anal Mach Intell 35, 8 (2013), 1798-1828.

[3] Emre Cakir, Toni Heittola, Heikki Huttunen, and Tuomas Virtanen. 2015. Poly phonic sound event detection using multi label deep neural networks. In International foint Conference on Neural Networks. IEEE, 1-7.

[4] Xi Chen, Yan Duan, Rein Houthooft, John Schulman, Ilya Sutskever, and Pieter Abbeel. 2016. Infogan: Interpretable representation learning by information maximizing generative adversarial nets. In Advances in Neural Information Processing Systems. 2172-2180.

[5] Brian Cheung, Jesse A Livezey, Arjun K Bansal, and Bruno A Olshausen. 2014 Discovering hidden factors of variation in deep networks. arXiv preprint arXiv:1412.6583 (2014)

[6] Selina Chu, Shrikanth Narayanan, C C Kuo, and Maja Mataric. 2006. Where am I? Scene recognition for mobile robots using audio features. In IEEE International Conference on Multimedia and Expo. IEEE, 885-888.

[7] Taco S Cohen and Max Welling. 2015. Transformation properties of learned visual representations. International Conference on Representation Learning (2015)

[8] S. Davis and P. Mermelstein. 1990. Comparison of parametric representations for monosyllabic word recognition in continuously spoken sentences. Readings in Speech Recognition 28, 4 (1990), 65-74.

[9] Arnaud Dessein, Arshia Cont, and Guillaume Lemaitre. 2013. Real-time detection of overlapping sound events with non-negative matrix factorization. In Matrix Information Geometry. Springer, 341-371.

[10] Frederic Font, Gerard Roma, and Xavier Serra. 2013. Freesound technical demo. In Proceedings of the 21st ACM international conference on Multimedia. ACM, 411-412.

[11] Oguzhan Gencoglu, Tuomas Virtanen, and Heikki Huttunen. 2010. Recognition of acoustic events using deep neural networks. In Signal Processing Conference. 506-510.

[12] Ian Goodfellow, Jean Pouget-Abadie, Mehdi Mirza, Bing Xu, David Warde-Farley, Sherjil Ozair, Aaron Courville, and Yoshua Bengio. 2014. Generative adversarial nets. In Advances in Neural Information Processing Systems. 2672-2680.

[13] Alex Graves. 2008. Supervised Sequence Labelling with Recurrent Neural Networks. Studies in Computational Intelligence 385 (2008).

[14] Aki Harma, Martin F McKinney, and Janto Skowronek. 2005. Automatic surveillance of the acoustic activity in our living environment. In IEEE International Conference on Multimedia and Expo. IEEE, 4-pp.

[15] Toni Heittola, Annamaria Mesaros, Tuomas Virtanen, and Moncef Gabbouj. 2013 Supervised model training for overlapping sound events based on unsupervised source separation.. In International Conference on Acoustics, Speech and Signal Processing. 8677-8681.

[16] Irina Higgins, Loic Matthey, Arka Pal, Christopher Burgess, Xavier Glorot, Matthew Botvinick, Shakir Mohamed, and Alexander Lerchner. 2017. Betavae: Learning basic visual concepts with a constrained variational framework. In International Conference on Learning Representations.

[17] G Hinton. 2008. Visualizing High-Dimensional Data Using t-SNE. Journal of Machnine Learning Research 9 (2008), 2579-2605.

[18] Satoshi Innami and Hiroyuki Kasai. 2012. NMF-based environmental sound source separation using time-variant gain features. Computers \& Mathematics with Applications 64, 5 (2012), 1333-1342.

[19] Diederik P Kingma, Tim Salimans, Rafal Jozefowicz, Xi Chen, Ilya Sutskever, and Max Welling. 2016. Improved variational inference with inverse autoregressive flow. In Advances in Neural Information Processing Systems. 4743-4751.
[20] Diederik P Kingma and Max Welling. 2014. Auto-encoding variational bayes. International Conference on Representation Learning (2014).

[21] Christian Kroos and Mark D. Plumbley. 2017. Neuroevolution for Sound Event Detection in Real Life Audio: A Pilot Study. In Detection \& Classification of Acoustic Scenes \& Events Workshop.

[22] Tejas D Kulkarni, William F. Whitney, Pushmeet Kohli, and Josh Tenenbaum. 2015. Deep Convolutional Inverse Graphics Network. In Conference and Workshop on Neural Information Processing Systems. 2539-2547.

[23] Daniel D Lee and H Sebastian Seung. 1999. Learning the parts of objects by non-negative matrix factorization. Nature 401, 6755 (1999), 788

[24] Yingzhen Li and Stephan Mandt. 2018. Disentangled Sequential Autoencoder. In Proceedings of the 35th International Conference on Machine Learning. 5670-5679.

[25] Zhuohan Li, Di He, Fei Tian, Wei Chen, Tao Qin, Liwei Wang, and Tieyan Liu. 2018. Towards Binary-Valued Gates for Robust LSTM Training. In Proceedings of the 35th International Conference on Machine Learning. 2995-3004.

[26] Ian McLoughlin, Haomin Zhang, Zhipeng Xie, Yan Song, and Wei Xiao. 2015. Robust sound event classification using deep neural networks. IEEE/ACM Transactions on Audio, Speech, and Language Processing 23, 3 (2015), 540-552.

[27] Annamaria Mesaros, Toni Heittola, Aleksandr Diment, Benjamin Elizalde, Ankit Shah, Emmanuel Vincent, Bhiksha Raj, and Tuomas Virtanen. 2017. DCASE 2017 challenge setup: Tasks, datasets and baseline system. In DCASE 2017-Workshop on Detection and Classification of Acoustic Scenes and Events.

[28] Annamaria Mesaros, Toni Heittola, Antti Eronen, and Tuomas Virtanen. 2010. Acoustic event detection in real life recordings. In Signal Processing Conference. IEEE, 1267-1271.

[29] Seongkyu Mun, Suwon Shon, Wooil Kim, and Hanseok Ko. 2016. Deep Neural Network Bottleneck Features for Acoustic Event Recognition.. In INTERSPEECH. 2954-2957.

[30] Giambattista Parascandolo, Heikki Huttunen, and Tuomas Virtanen. 2016. Recurrent neural networks for polyphonic sound event detection in real life recordings. International Conference on Acoustics, Speech, and Signal Processing (2016), 64406444.

[31] Scott Reed, Kihyuk Sohn, Yuting Zhang, and Honglak Lee. 2014. Learning to disentangle factors of variation with manifold interaction. In International Conference on Machine Learning. 1431-1439.

[32] Manjeet Rege, Ming Dong, and Farshad Fotouhi. 2008. Bipartite isoperimetric graph partitioning for data co-clustering. Data Mining and Knowledge Discovery 16,3 (2008), 276-312.

[33] Li Wan, Matthew Zeiler, Sixin Zhang, Yann Le Cun, and Rob Fergus. 2013. Regularization of Neural Networks using DropConnect. In Proceedings of the 30th International Conference on Machine Learning. 1058-1066.

[34] Yun Wang and Florian Metze. 2017. A Transfer Learning Based Feature Extractor for Polyphonic Sound Event Detection Using Connectionist Temporal Classification. In INTERSPEECH. 3097-3101.

[35] Xianjun Xia, Roberto Togneri, Ferdous Sohel, and David Huang. 2017. FrameWise Dynamic Threshold Based Polyphonic Acoustic Event Detection. In INTERSPEECH.

[36] Jimei Yang, Scott E Reed, Minghsuan Yang, and Honglak Lee. 2015. Weaklysupervised disentangling with recurrent transformations for $3 \mathrm{~d}$ view synthesis. In Advances in Neural Information Processing Systems. 1099-1107.

[37] Dongqing Zhang and Dan Ellis. 2001. Detecting sound events in basketball video archive. Department of Electrical Engineering, Columbia University, New York (2001).

[38] Matthias Zhrer and Franz Pernkopf. 2017. Virtual Adversarial Training and Data Augmentation for Acoustic Event Detection with Gated Recurrent Neural Networks. In INTERSPEECH. 493-497. 